\title{
A Case of Mucous Membrane Pemphigoid: From Diagnosis to Therapeutic Strategy
}

\author{
Kun-Hwa Kang, Jin-Seok Byun, Jae-Kwang Jung, Jae-Kap Choi
}

\author{
Department of Oral Medicine, School of Dentistry, Kyungpook National University, Daegu, Korea
}

Received May 25, 2021

Revised June 11, 2021

Accepted June 11, 2021
Correspondence to:

Jae-Kap Choi

Department of Oral Medicine, School of Dentistry, Kyungpook National University,

2177 Dalgubeol-daero, Jung-gu, Daegu 41940, Korea

Tel: $+82-53-600-7321$

Fax: +82-53-426-2195

E-mail: jhchoi@knu.ac.kr

https://orcid.org/0000-0001-6773-7507
Mucous membrane pemphigoid (MMP) is a heterogeneous group of chronic, autoimmune subepithelial blistering diseases, predominantly involving oral mucosal membrane. Because of its pathophysiology of autoimmune reaction, MMP-related gingivitis would not respond to conventional periodontal treatments. We present a case of a 65 -year-old female with a chief complaint of chronic generalized buccal gingivitis, unimproved after periodontal treatment for four months. Based on the clinical findings, histological examination, and direct immunofluorescence microscopy, it was diagnosed as MMP. The symptoms were relieved with immunomodulatory therapy using corticosteroids and the supportive management of professional plaque control. MMP can cause pathological involvement throughout the oral and other mucosae of the body, thus leading to functional impairment through repeated inflammatory cascades. Therefore, accurate diagnosis is essential to properly manage local and possible systemic complications of MMP.

Key Words: Gingivitis; Mucous membrane pemphigoid; Subepithelial autoimmune disease; Topical corticosteroids

\section{INTRODUCTION}

Mucous membrane pemphigoid (MMP) is a heterogeneous group of chronic autoimmune subepithelial blistering diseases, which can be potentially life-threatening when it occurs at laryngeal or esophageal areas [1,2]. It can affect single or multiple sites including oral, ocular, genital, nasopharyngeal, esophageal, laryngeal mucosa, and skin [3]. The affected mucosa or skin manifests with blistering, erythema, erosion, and subsequent "scarring" as the cascade of autoinflammatory reactions [2]. Furthermore, the subsequent mucosal scarring in the upper airway and eye could lead to airway obstruction and blindness, respectively [3].

The annual incidence of MMP was reported to be 1.3 and 2.0 per 1 million people in France and Germany, respectively [4]. MMP mainly occurs in elderly adults with ages between 60 and 80 years [5]. Females are predominantly affected compared to males with a female-to-male ratio of 2.27:1 [6].

Although the MMP etiology remains to be clearly established, autoantibodies against hemidesmosomes of the basement membrane zone (BMZ) could play a role in the pathogenesis of the disease [3]. The enzyme-linked immunosorbent assay (ELISA) and immunoblotting or immunoprecipitation methods were used to detect a number of autoantigens, including bullous pemphigoid antigen 1 (BPAg1 and BP230), bullous pemphigoid antigen 2 (BPAg2 and BP180) [7,8], laminin-5 (epiligrin and laminin-332) [9], laminin-6 [10], type VII collagen [11], and integrin $\alpha-6 /$ $\beta$-4 subunit [12]. Furthermore, both autoantibodies and cellular immunity were reported to be possible initiating factors of subepithelial cleft [3]. The binding of autoantibodies to those autoantigens within the epithelial BMZ could activate the T cells and complement the cascade [13]. The

Copyright (c) 2021 Korean Academy of Orofacial Pain and Oral Medicine. All rights reserved.

(c) This is an open-access article distributed under the terms of the Creative Commons Attribution Non-Commercial License (http://creativecommons.org/licenses/by-nc/4.0/), which permits unrestricted non-commercial use, distribution, and reproduction in any medium, provided the original work is properly cited. 
accumulation of complement 3 (C3) fragments within lamina lucida results in vasodilatation and recruitment of inflammatory cells, subsequently causing tissue damage by the released proinflammatory cytokines and proteinases [13]. In the direct immunofluorescence (DIF) microscopy, it is characterized by continuous deposition of any single or combination of immunoglobulin G (IgG), immunoglobulin A (IgA), or C3 along the BMZ, thus disturbing the cellular junction and histostructural integrity of the BMZ.

The subepithelial detachment caused by the disturbed cellular junction would result in the formation of blisters and subsequent ulceration, which could be often confused with those attributed to bacterial gingivitis and other mucosal diseases, including desquamative lichen planus (LP), erythema multiforme (EM), and pemphigus vulgaris (PV) due to similar clinical manifestation [2]. However, the symptoms of gingival inflammation of MMP could not be improved just by the conventional application of antibiotics or subgingival curettage for chronic gingivitis because of the difference between their underlying pathogenesis. Therefore, biopsy should be considered to make an accurate differential diagnosis if chronic gingivitis with desquamation does not respond to conventional periodontal treatments. In this study, we report a case of a patient who was referred to the department of oral medicine because of refractory inflammation on marginal gingiva, initially misdiagnosed and treated as chronic gingivitis.

\section{CASE REPORT}

A 65-year-old female complaining of generalized gingival bleeding and soreness from about 4 months ago was referred to the Department of Oral Medicine of the Kyungpook National University Dental Hospital from the Department of Periodontology at the same hospital. Her medical history revealed that she had been taking valsartan, an angiotensin II receptor antagonist, for hypertension management for three years.

The clinical examination revealed irregular ulceration with a yellow base, surrounded by the fine erythematous border along the free gingival line (Fig. 1). However, there was neither loss of alveolar bone nor accumulation of calculus, suggestive of periodontal diseases on panoramic radiographs. Routine laboratory work-up, including complete blood count (CBC), C-reactive protein, and antinuclear antibodies, was within the normal range. Additionally, the dental history revealed that her signs and symptoms were not resolved even with repetitive periodontal treatments for four months.

Incisional biopsies were performed on two separated areas of the left mandibular buccal gingiva, one for conventional histopathological evaluation and the other for immunofluorescence examination. The epithelial layer was observed to be easily peeled off from the base of the labial gingiva, whereas the tissue was slightly pulled out for biopsy, implying a positive Nikolsky sign (Fig. 2). The histopathological examination showed the subepithelial split and focal infiltration of inflammatory cells along the basement membrane (Fig. 3). The DIF examination also revealed linear accumulation of IgG and C3 along the basal lamina. These histopathological and immunofluorescence findings were consistent with the histopathological characteristics
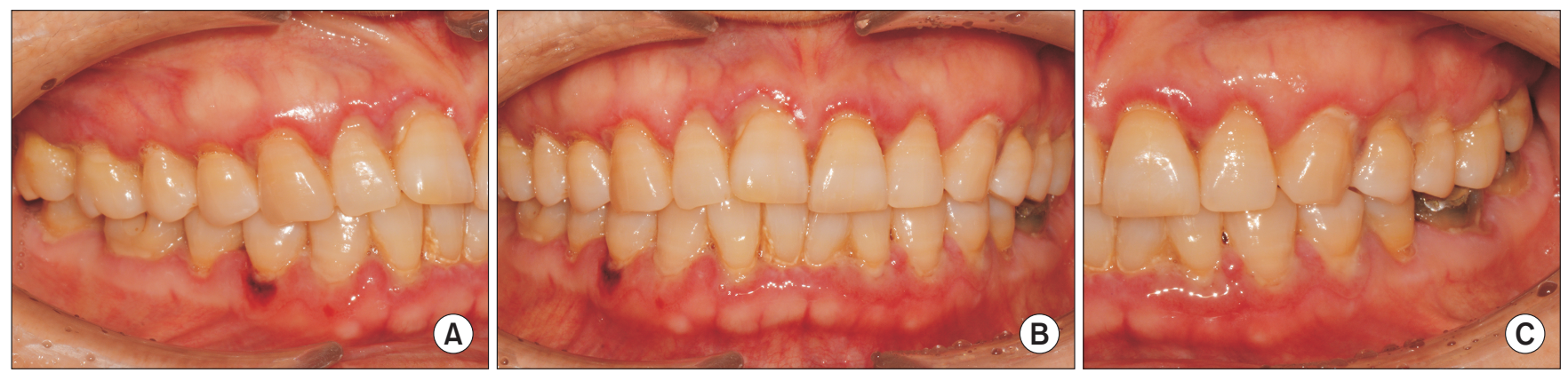

Fig. 1. Clinical photographs showing desquamative buccal gingiva at the first visit. (A) Bleeding on \#44 buccal attached gingiva. (B) Irregular ulcerations with a yellow base surrounded by the fine erythematous border along the free gingival line. (C) Gingival swelling and blistering present on the left mandibular anterior buccal gingiva. 
of MMP. Based on clinical and histopathological findings, the diagnosis of MMP was established. Ophthalmologist and gynecologist consultation was performed to determine the possible involvement on other mucosal regions. No further involvement was confirmed through the medical examinations.

Topical and systemic corticosteroids were applied as initial

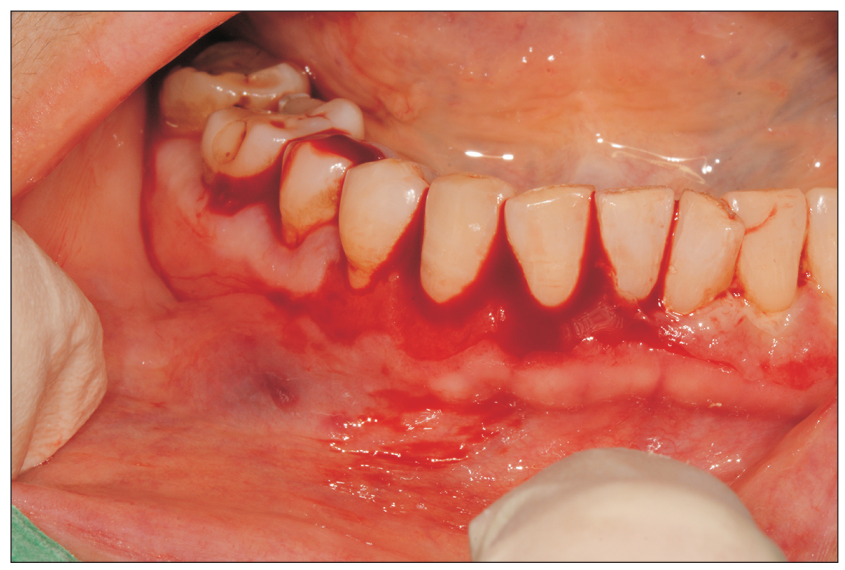

Fig. 2. Positive Nikolsky sign on the right mandibular buccal mucosa. therapy for several weeks. However, the painful gingivitis was sustained with no visible improvement. Furthermore, the lesion was significantly improved after four weeks of immunosuppressive therapy with azathioprine $100 \mathrm{mg}$ per day (Fig. 4). However, the patient complained of alopecia, and the follow-up laboratory test showed leukopenia. Thus, azathioprine administration was immediately stopped. For the improvement in topical drug delivery, the custom-made acrylic trays were prepared for maxilla and mandible using a thermoforming sheet and then coated on the inside with 0.05\% fluocinonide ointment (Fig. 5A). Additionally, professional plaque control was regularly performed to minimize the plaque-induced inflammation that could be coupled with the autoimmune response. The symptoms were relieved without any side effects after topical steroid application using tray (Fig. 5B).

\section{DISCUSSION}

The most frequent site of MMP is the oral mucosa (85\% of patients), followed by ocular (65\%), nasal (20\%-40\%),
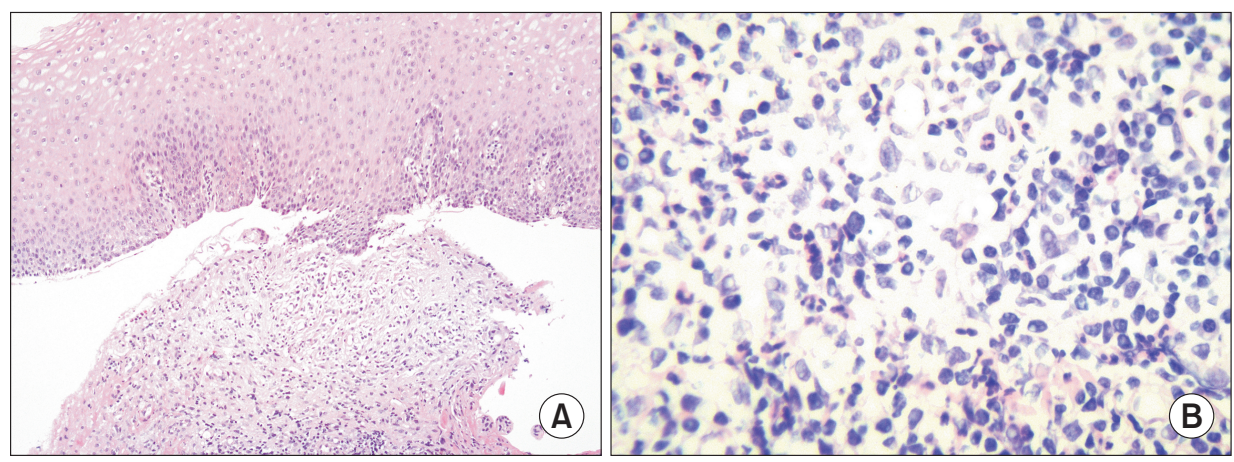

Fig. 3. Histopathological findings of the right mandibular buccal gingiva. (A) Separation of epithelium (H\&E staining, $\times 200$ magnification). (B) Infiltration of various immune cells (H\&E staining, $x$ 400 magnification).
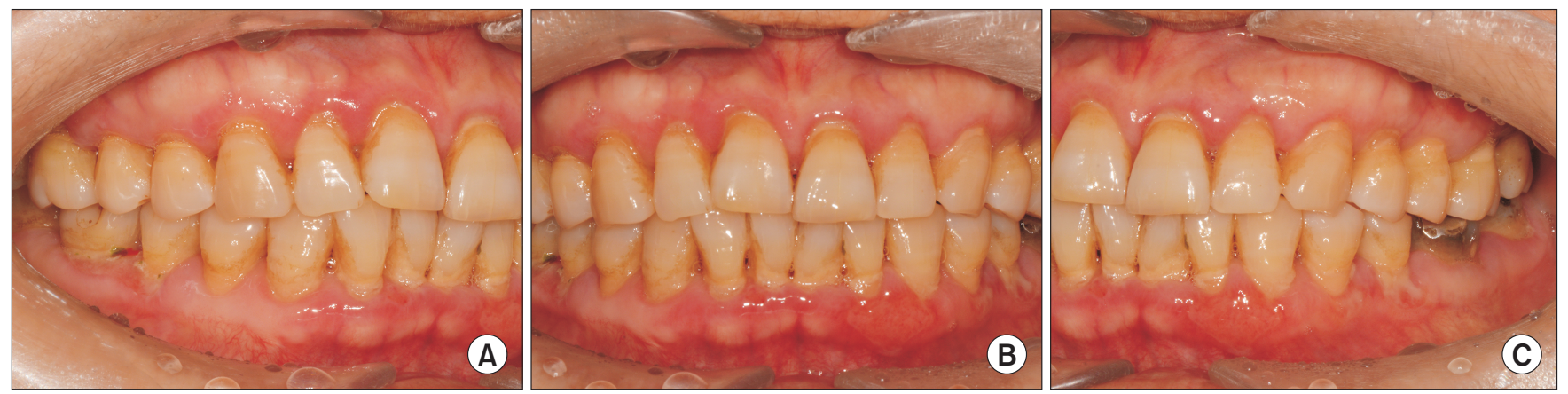

Fig. 4. Clinical photographs showing improvement 4 weeks after azathioprine. (A) Mild gingival swelling with redness on the right maxillary and mandibular anterior buccal gingiva. (B) The previous erythematous border and irregular ulceration resolved. (C) Recently formed erosive lesion observed on \#35 buccal attached gingiva. 

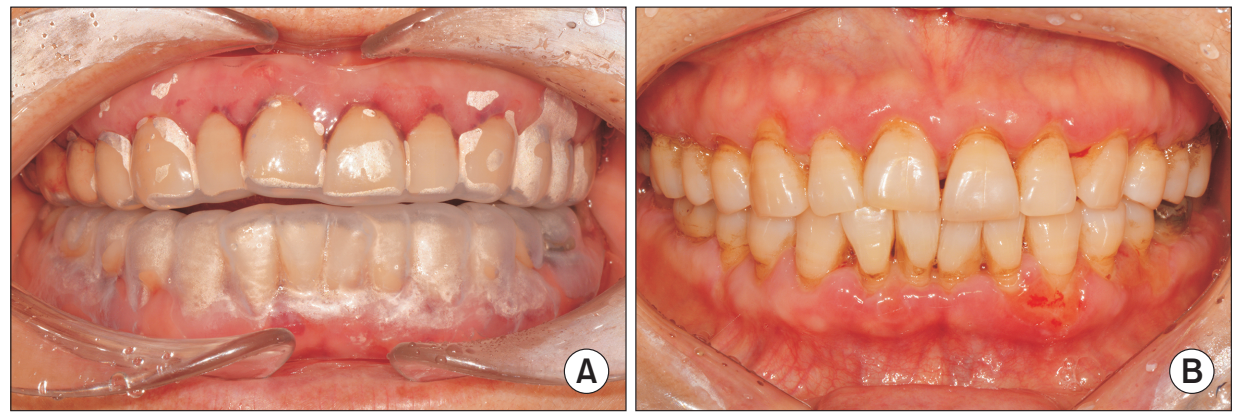

Fig. 5. (A) Custom-made acrylic trays coated on the inside with $0.05 \%$ fluocinonide ointment. (B) Clinical photographs after the application of topical steroid using trays and regular professional plaque control.

skin (25\%-30\%), anogenital (20\%), pharyngeal (20\%), laryngeal (5\%-15\%), and esophageal (5\%-15\%) mucosa [14]. Also, the most common site of initial involvement of MMP is the oral mucosa [15]. Among the intraoral sites, the most commonly involved site is gingiva (80\%), followed by buccal mucosa (58\%), palate (26\%), alveolar ridge (16\%), tongue (15\%), and lower lip (7\%) [16].

The most characteristic feature in oral MMP is desquamative gingivitis [15]. Desquamative gingivitis could be caused by allergies as well as other autoimmune diseases, including erosive LP, PV, and EM [17]. It is difficult to distinguish erosive LP from other autoimmune bullous diseases (AIBDs) based on the clinical findings, despite some characteristic differences. Another clinical characteristic of MMP is the positive Nikolsky sign, an easy desquamation of the epithelium by slight sliding pressure, but not common in LP [18]. However, the Nikolsky sign was considered a valid but not highly reliable clinical feature for the differential diagnosis of MMP because it could be often false-negative for MMP but might also be positive for other AIBDs [19].

A wide range of clinical presentations could be manifested among patients with MMP. On one hand, "low-risk" patients are defined as those who have the disease affecting only the oral mucosa and/or the skin with less susceptibility to scarring. On the other hand, "high-risk" patients are those with any further involvement on the critical sites including ocular, nasopharyngeal, esophageal, laryngeal, and/ or genital mucosa [1]. The great tendency to scarring on these critical sites could be linked with serious and sometimes fatal conditions including blindness, asphyxiation, dysphagia, urethral strictures, and/or vaginal stenosis [2]. Furthermore, therapeutic options to prevent scarring might be limited once the autoimmune reactions would progress to the apparent inflammatory condition. Therefore, it was emphasized that early and accurate diagnosis would play a critical role in maintaining the oral and systemic health of patients with MMP [3]. However, the rarity of MMP and its nonspecific clinical presentation often leads to misdiagnoses and inappropriate treatment [18].

Accordingly, besides the clinical examination, histopathological and immunopathological tests should be considered to establish an accurate diagnosis [20]. Typical MMP histopathology exhibits the subepithelial cleft and infiltration of inflammatory cells in its vicinity [18]. Based on these histological findings, MMP can usually be differentiated from intraepithelial autoimmune blistering dermatoses, including LP, PV, and EM, characterized by the blister formation in the suprabasilar portion of the oral mucosa. However, it is difficult to distinguish MMP from other subepithelial blistering dermatoses (SEBDs), such as bullous pemphigoid, epidermolysis bullosa acquisita, or bullous systemic lupus erythematosus (BSLE) [2]. As a result of this, DIF is also considered another gold standard of diagnostic tests for MMP, sensitivity of which lies between 69\% and 83\% [1]. However, neither routine histology nor DIF is solely sufficient to distinguish MMP from other SEBDs [2]. Therefore, the comprehensive consideration of clinical features, histological findings, and DIF examination is ultimately recommended for the confirmative diagnosis of MMP [2].

Additionally, immunoserological tests, such as indirect immunofluorescence (IIF) microscopy, ELISA, and immunoblot assay, are also occasionally performed. Although serum circulating autoantibodies are usually low in MMP, IIF is useful for identifying isotype and binding pattern of the circulating anti-BMZ antibodies. Laminin-5, laminin-6, and type VII collagen autoantibodies bind to the dermal side, whereas BPAg2 and $\alpha-6 / \beta-4$ integrins autoantibodies bind to the epidermal side [2]. Target antigens with circulating 
autoantibodies are specified using ELISA and immunoblot assays. However, ELISA in nonpemphigoid controls shows a high number of false-positive results (11.3\%) [21]. The immunoblot assay also requires highly sophisticated preparation of dermal samples and has a low sensitivity [22].

The management goal of MMP is to conserve function and to prevent the irreversible scarring process [2]. There is a first international consensus of treatment guidelines of MMP, dividing patients into "low-risk" and "high-risk" groups, according to the involved sites and clinical severity [1]. For the "low-risk" group, topical corticosteroid is usually recommended as a first choice [1]. It includes topical betamethasone, 0.05\% clobetasol propionate ointment, and intralesional injection of triamcinolone [2]. Successful treatment with dapsone was also reported in patients with oral and ocular diseases [23]. Additionally, a low dose of systemic corticosteroids and immunosuppressives, such as azathioprine or mycophenolate mofetil, could be added with topical corticosteroids [24].

For the "high-risk" group, dapsone, prednisone, azathioprine, mycophenolate mofetil, and cyclophosphamide can be prescribed [1]. However, azathioprine and mycophenolate mofetil may induce liver toxicity, cyclophosphamide may cause hemorrhagic cystitis, and severe bone marrow suppression can be incurred by any of these immunosuppressive agents. Therefore, laboratory monitoring should be performed before and during treatment [25].

Recently, it has been shown that anti-CD20 antibody (rituximab), intravenous immunoglobulin (IVIG), and combination therapy of both biological agents are effective for resistant MMP [26-28]. They are highly promising alternatives. However, there has been no long-term monitoring.

Patients with MMP are known to be more vulnerable to chronic periodontitis because erosive gingival lesions may produce a proper environment for bacterial growth [29]. Additionally, periodontal inflammatory processes could trigger and exacerbate an autoimmune response [30]. Therefore, dental practitioners should consider regular plaque control as the adjunctive therapeutic strategy.

In conclusion, MMP occurs most frequently and initially in the oral mucosa, especially in the gingiva. If the pathological lesion of MMP presents only in the oral mucosa, early and accurate diagnosis is difficult because MMP is rare and the symptoms are similar to bacterial gingivitis. However, early diagnosis and appropriate treatment are important for the prevention of possible complications and the subsequent maintenance of function in patients with MMP. Therefore, it is important to refer patients with refractory desquamative gingivitis to an oral medicine specialist considering the non-plaque-induced gingival diseases. Oral medicine specialists have to provide an individualized therapeutic strategy for the management of MMP considering the variety of disease conditions.

\section{CONFLICT OF INTEREST}

No potential conflict of interest relevant to this article was reported.

\section{ORCID}

\author{
Kun-Hwa Kang \\ https://orcid.org/0000-0003-0140-4501 \\ Jin-Seok Byun \\ https://orcid.org/0000-0002-6182-1238 \\ Jae-Kwang Jung \\ https://orcid.org/0000-0003-3099-8097 \\ Jae-Kap Choi \\ https://orcid.org/0000-0001-6773-7507
}

\section{REFERENCES}

1. Chan LS, Ahmed AR, Anhalt GJ, et al. The first international consensus on mucous membrane pemphigoid: definition, diagnostic criteria, pathogenic factors, medical treatment, and prognostic indicators. Arch Dermatol 2002;138:370-379.

2. Xu HH, Werth VP, Parisi E, Sollecito TP. Mucous membrane pemphigoid. Dent Clin North Am 2013;57:611-630.

3. Chan LS. Mucous membrane pemphigoid. Clin Dermatol 2001;19:703-711

4. Bertram F, Bröcker EB, Zillikens D, Schmidt E. Prospective analysis of the incidence of autoimmune bullous disorders in Lower Franconia, Germany. J Dtsch Dermatol Ges 2009;7:434-440.

5. Ahmed AR, Kurgis BS, Rogers RS 3rd. Cicatricial pemphigoid. J Am Acad Dermatol 1991;24(6 Pt 1):987-1001.

6. Ahmed AR, Hombal SM. Cicatricial pemphigoid. Int J Dermatol 1986;25:90-96.

7. Bernard P, Prost C, Lecerf V, et al. Studies of cicatricial pemphigoid autoantibodies using direct immunoelectron microscopy and immunoblot analysis. J Invest Dermatol 1990;94:630-635. 
8. Balding SD, Prost C, Diaz LA, et al. Cicatricial pemphigoid autoantibodies react with multiple sites on the BP180 extracellular domain. J Invest Dermatol 1996;106:141-146.

9. Kirtschig G, Marinkovich MP, Burgeson RE, Yancey KB. Antibasement membrane autoantibodies in patients with anti-epiligrin cicatricial pemphigoid bind the alpha subunit of laminin 5. J Invest Dermatol 1995;105:543-548.

10. Chan LS, Majmudar AA, Tran HH, et al. Laminin-6 and laminin-5 are recognized by autoantibodies in a subset of cicatricial pemphigoid. J Invest Dermatol 1997;108:848-853.

11. Luke MC, Darling TN, Hsu R, et al. Mucosal morbidity in patients with epidermolysis bullosa acquisita. Arch Dermatol 1999;135:954-959.

12. Tyagi S, Bhol K, Natarajan K, Livir-Rallatos C, Foster CS, Ahmed AR. Ocular cicatricial pemphigoid antigen: partial sequence and biochemical characterization. Proc Natl Acad Sci U S A 1996;93:14714-14719.

13. Georgoudis P, Sabatino F, Szentmary N, et al. Ocular mucous membrane pemphigoid: current state of pathophysiology, diagnostics and treatment. Ophthalmol Ther 2019;8:5-17.

14. Schmidt E, Zillikens D. Pemphigoid diseases. Lancet 2013;381:320-332.

15. Scully C, Lo Muzio L. Oral mucosal diseases: mucous membrane pemphigoid. Br J Oral Maxillofac Surg 2008;46:358-366.

16. Bagan J, Jiménez Y, Murillo J, Bagan L. Oral mucous membrane pemphigoid: a clinical study of 100 low-risk cases. Oral Dis 2018;24:132-134.

17. Al-Abeedi F, Aldahish Y, Almotawa Z, Kujan 0. The differential diagnosis of desquamative gingivitis: review of the literature and clinical guide for dental undergraduates. J Int Oral Health 2015;7(Suppl 1):88-92.

18. Buonavoglia A, Leone P, Dammacco R, et al. Pemphigus and mucous membrane pemphigoid: an update from diagnosis to therapy. Autoimmun Rev 2019;18:349-358.

19. Uzun S, Durdu M. The specificity and sensitivity of Nikolskiy sign in the diagnosis of pemphigus. J Am Acad Dermatol 2006;54:411-
415.

20. Rashid H, Lamberts A, Diercks GFH, et al. Oral lesions in autoimmune bullous diseases: an overview of clinical characteristics and diagnostic algorithm. Am J Clin Dermatol 2019;20:847-861.

21. Meijer JM, Diercks GFH, de Lang EWG, Pas HH, Jonkman MF. Assessment of diagnostic strategy for early recognition of bullous and nonbullous variants of pemphigoid. JAMA Dermatol 2019;155:158-165.

22. Goletz S, Hashimoto T, Zillikens D, Schmidt E. Anti-p200 pemphigoid. J Am Acad Dermatol 2014;71:185-191.

23. Miserocchi E, Baltatzis S, Roque MR, Ahmed AR, Foster CS. The effect of treatment and its related side effects in patients with severe ocular cicatricial pemphigoid. Ophthalmology 2002;109:111118.

24. Chan LS. Ocular and oral mucous membrane pemphigoid (cicatricial pemphigoid). Clin Dermatol 2012;30:34-37.

25. Neff AG, Turner M, Mutasim DF. Treatment strategies in mucous membrane pemphigoid. Ther Clin Risk Manag 2008;4:617-626.

26. Amagai M, Ikeda S, Shimizu H, et al. A randomized double-blind trial of intravenous immunoglobulin for pemphigus. J Am Acad Dermatol 2009;60:595-603.

27. Foster CS, Chang PY, Ahmed AR. Combination of rituximab and intravenous immunoglobulin for recalcitrant ocular cicatricial pemphigoid: a preliminary report. Ophthalmology 2010;117:861869.

28. Ahmed AR, Shetty S. A comprehensive analysis of treatment outcomes in patients with pemphigus vulgaris treated with rituximab. Autoimmun Rev 2015;14:323-331.

29. Schellinck AE, Rees TD, Plemons JM, Kessler HP, Rivera-Hidalgo F, Solomon ES. A comparison of the periodontal status in patients with mucous membrane pemphigoid: a 5-year follow-up. J Periodontol 2009;80:1765-1773.

30. Jascholt I, Lai O, Zillikens D, Kasperkiewicz M. Periodontitis in oral pemphigus and pemphigoid: a systematic review of published studies. J Am Acad Dermatol 2017;76:975-978.e3. 